\title{
INFECÇÃO BACTERIANA NO TRANSPLANTE INTESTINAL E MULTIVISCERAL - REVISÃO DA LITERATURA
}

\author{
Bacterial infection in Small Bowel and Multivisceral Transplantation - Literature Review
}

\author{
Adriana Weinfeld Massaia', Catiana Mitica Gritti ${ }^{2}$, André Ibrahim David ${ }^{3}$, Ivan Leonardo Avelino França e Silva ${ }^{4}$, Edson Abdala ${ }^{5}$
}

\section{RESUMO}

A natureza das infecções após transplantes de órgãos sólidos mudou, significativamente, com o advento de potentes esquemas imunossupressores, o uso rotineiro de profilaxia antimicrobiana e os avanços das técnicas de diagnóstico microbiológico. Novos patógenos estão sendo identificados nessa população, incluindo vários com significante perfil de resistência a antimicrobianos. A cronologia das infecções após transplante é determinada pela natureza e intensidade das exposições epidemiológicas individuais e do estado de equilíbrio da imunossupressão. A escassez de estudos epidemiológicos das infecções pós-transplantes de intestino e multiviscerais ainda mantém sua compreensão mais no estado da arte do que como ciência. O objetivo deste estudo é descrever os dados epidemiológicos das infecções bacterianas pós-transplantes de intestino e multiviscerais. Os agentes mais prevalentes nos transplantes de intestino e multiviscerais foram: Enterococcus spp., Staphylococcus spp., Streptococcus spp., Pseudomonas aeruginosa, Klebsiella pneumoniae, Escherichia coli, Enterobacter spp. Os principais sítios de infecção foram: sítio cirúrgico, intra-abdominal, corrente sanguínea, cateter venoso central, urinário e respiratório. Os fatores de risco identificados foram: rejeição aguda, dose alta de imunossupressão, uso de dispositivos invasivos, cirurgias extensas e reoperação, enxerto contaminado, infecção prévia e paciente hospitalizado no período pré-transplante.

Descritores: Infecções Bacterianas; Intestino Delgado; Transplante.

Instituições:

${ }^{1}$ Departamento de Infectologia da Santa Casa de São Paulo, São Paulo/SP

${ }^{2}$ Gestão de Transplante de Fígado da Beneficência Portuguesa de São Paulo, São Paulo/SP

${ }^{3}$ Faculdade de Medicina da Universidade de São Paulo, Departmento de Gastroenterologia, Programa de Transplante Hepático e Gastrointestinal, (LIM 37), São Paulo/SP,

${ }^{4}$ Departamento de Infectologia e SCIH do Hospital A.C.Camargo, São Paulo/SP

${ }^{5}$ Departamento de Infectologia do Hospital das Clínicas da FMUSP, São Paulo/SP

\section{Correspondência:}

Adriana Weinfeld Massaia

End.: Rua Itapirapuã, 278 - CEP 01440-040 - São Paulo / SP

Tel: 5511 98199-7378

E-mail: dri.weinfeld@gmail.com

Recebido em: 06/05/2013

Aceito em: 28/06/3013

\section{INTRODUÇÃO}

As complicações infecciosas são umas das maiores preocupações nos pacientes que se submeteram ao Transplante de Intestino (TI) e ao Transplante Multivisceral (TMV), uma vez que se inserem nas principais causas de morbidade e mortalidade dessas modalidades de transplante. Segundo alguns estudos, 58 a $80 \%$ dos pacientes apresentam ao menos um episódio de infecção no primeiro mês após o transplante. Em uma série de casos, a infecção foi documentada em $76,2 \%$, com mortalidade de $17,8 \%{ }^{1}$ A história natural das infecções após o transplante é determinada pela natureza e intensidade da exposição epidemiológica individual e pelo estado de equilíbrio da imunossupressão. ${ }^{2}$

Infecções bacterianas ocorrem em 33 a 54\% dos receptores de Transplante de Órgãos Sólidos (TOS). Há relatos de incidência maior do que $93 \%$ no TI. O tipo de infecção bacteriana está relacionado ao tipo de TOS, tempo de internação hospitalar, gravidade do paciente e tratamento imunossupressor. A principal causa de infecção no primeiro mês após o TOS é infecção bacteriana, principalmente a adquirida no ambiente hospitalar. ${ }^{3-5}$ 
Poucos estudos demonstram os fatores de risco pré-transplante. Goulet e colaboradores reportaram pior evolução clínica quando havia múltiplas cirurgias abdominais prévias, sepse por fungo, rejeição resistente a corticosteróide, hospitalização prolongada antes do transplante, retransplante, trombose de veia cava inferior e pseudobstrução intestinal. ${ }^{6}$ Abu-Elmaged e colaboradores, em sua experiência com 500 TI, observaram que os resultados melhoravam de acordo com períodos curtos de NPT, idade, era de imunossupressão, tipo de enxerto (fígado-intestino) e imunossupressão. ${ }^{7}$ Kato e colaboradores constataram alguns significantes preditores de baixa sobrevida como tipo de enxerto (intestino isolado e fígado-intestino), terapia de indução (ausência de anti-IL-2), hospitalização prolongada antes do transplante e idade. ${ }^{8}$ Segundo o Intestine Transplant Registry 2009, a sobrevida era influenciada pela era da imunossupressão, volume administrado, status prétransplante e transplante de fígado combinado. ${ }^{9}$ Farmer e colaboradores relataram, em um estudo com 88 pacientes e $106 \mathrm{TI}$, que anticorpo específico do doador, inclusão do transplante de fígado e esplenectomia do receptor eram fatores independentes de sobrevida. Melhores resultados eram vistos quando o receptor não portava anticorpo contra o doador, não realizava esplenectomia e era submetido ao transplante combinado de fígado. ${ }^{9}$

O risco de infecção varia conforme o tempo de progressão após transplante, tipo e intensidade de imunossupressão e grau de exposição aos diferentes micro-organismos. No entanto, coleções pós-operatórias, uso de drenos, isquemia tecidual, neutropenia, doenças crônicas associadas, disfunção orgânica, distúrbios metabólicos, exposição antimicrobiana, entre outros, também contribuem para o risco de adquirir infecção. ${ }^{2,3,10}$

Alguns fatores de risco associados já estão bem estabelecidos. São estes: procedimento cirúrgico, uso de dispositivos invasivos, hospitalização em Unidades de Terapia Intensiva (UTI) e exposição a antibióticos de amplo espectro, o que facilita também a aquisição de infecções por germes resistentes. A resistência antimicrobiana tem aumentado significativamente nessa população, devendo ser considerada na escolha da terapia antimicrobiana empírica. ${ }^{3,4,11,12}$

As infecções bacterianas são comumente causadas por micro-organismos que colonizam o paciente. Um mecanismo importante é a translocação bacteriana, facilitada pela perda da barreira intestinal. A translocação bacteriana, definida como a passagem de bactérias viáveis ou não e/ou seus produtos através do lúmen intestinal para locais estéreis, está cada vez mais associada aos mecanismos responsáveis pela infecção bacteriana após TI e TMV., ${ }^{3,413}$

\section{OBJETIVO}

Descrever os dados epidemiológicos das infecções pós-transplante de intestino e multiviscerais quanto à incidência, distribuição, fatores de risco e evolução, segundo revisão da literatura.

\section{MATERIAL E MÉTODO}

Foi realizada revisão de literatura através de busca no Medline/PubMed, Latin-American and Caribbean Centre on Health Sciences Information (Lilacs), Scientific Electronic Library Online (Scielo), Embase Biomedica Answers, Thomson Reuters (ISI) Web of Knowledge e SciVerseScopus. Os termos utilizados foram: "intestine transplantation", "intestine transplant”, "intestinal transplantation", "intestinal transplant", "multivisceral transplantation", "small bowel transplantation", "infectious transplantation", "intest ${ }^{*}$ transplant ${ }^{*}$, "small bowel transplant*" "multivisceral transplant", "infection transplantation", "bacterial infection transplant*", "viral infection transplant*", "fungal infection transplant" . Não se utilizou filtro. O período de revisão foi a partir do ano de 2000 até julho de 2013.

Os seguintes artigos foram selecionados para compor os dados de revisão:

A. Artigos originais que tratavam de TI ou TMV, e uma das seguintes situações: descrições de casuísticas com resultados e complicações, incluindo infecciosas; relatos ou estudos de infecções.

B. Artigos de revisão sobre TI ou TMV que incluíam informação sobre infecção.

C. Artigos originais ou de revisão em TOS que contivessem dados de infecção em TI ou TMV.

\section{ANÁLISE DE RESULTADO}

Pesquisadores da Universidade de Pittsburgh avaliaram a translocação bacteriana em 50 pacientes após TI, Transplante Fígado-Intestino (TFI) e TMV. Desses, 22 pacientes apresentaram translocação bacteriana, com as seguintes distribuições: 5/14 no TI, $14 / 28$ no TFI e 3/8 no TMV, sendo que o número de episódios foi de 8,29 e 4, respectivamente. Ocorreu predominantemente nos primeiros 30 dias após o transplante. A presença do cólon nos TI e TFI foi associada a $100 \%$ de supercrescimento bacteriano, além de maiores taxas de translocação bacteriana quando comparadas aos transplantes sem o cólon.14 Tempo de isquemia maior do que nove horas, também foi fator predisponente. ${ }^{15}$

Com o objetivo de identificar a possibilidade de antibioticoterapia guiada por coprocultura, autores do Mount Sinai Medical Center realizaram coprocultura, hemocultura, cultura peritoneal e de ferida operatória em 31 pacientes submetidos a TI ou TFI. Desses, 16 pacientes tiveram coprocultura positiva e, nestes, foram positivas sete hemoculturas, sete culturas de líquido peritoneal e sete de ferida operatória. Dos 17 pacientes que não tiveram 
coprocultura realizada, seis apresentaram hemoculturas positivas, 11 culturas de líquido peritoneal e quatro de ferida operatória. Os agentes isolados nas coproculturas foram Enterobacter aerogenes (E.aerogenes), Enterococcus faecalis (E.faecalis) e faecium (E.faecium), P.aeruginosa, Staphylococcus epidermidis (S.epidermidis), Citrobacter koseri, Klebsiella pneumoniae (K.pneumonia) e Escherichia coli (E.coli). Observou-se que os micro-organismos isolados em coprocultura não eram os mesmos isolados das infecções nos outros sítios (sangue, líquido peritoneal e ferida operatória). ${ }^{16}$
Recentemente, trabalhos publicados vêm demonstrando redução na incidência de infecções bacterianas por Grampositivos nos TOS, apesar de permanecer elevada quando se analisam os germes isoladamente. ${ }^{11,17}$ A prevalência de bactérias Gram-negativas MDR continua aumentando, bem como seu perfil de resistência. ${ }^{12,17}$ Observa-se também alta mortalidade das infecções causadas por patógenos MDR. ${ }^{12}$ Nos TI, TFI e TMV os dados são discordantes, não ocorrendo uma predominância clara de um dos grupos de agentes (Tabela 1). ${ }^{5,16,18-23}$

Tabela 1: Infecções por bactérias Gram-positivas e Gram-negativas nos transplantes de intestino e multiviscerais.

\begin{tabular}{|c|c|c|c|}
\hline Autor/ Origem/ Ano & $\begin{array}{c}\text { No total de pacientes / } \\
\text { No total de episódios de infecção }\end{array}$ & Agentes GP $\dagger$ & Agentes GN $\dagger$ \\
\hline $\begin{array}{l}\text { Akhter K et al/ Washington/ } \\
2012 .{ }^{5}\end{array}$ & $56 / 85^{\star}$ & $\begin{array}{l}\text { 56/85 (65,9\%) } \\
\text { Enterococcus spp.: } 17 \text { SCN: } 7 \\
\text { MRSA: } 2 \\
\text { S.viridans: } 5\end{array}$ & $\begin{array}{l}\text { 29/85 }(34,1 \%) \\
\text { Pseudomonas spp.: } 5 \text { Enterobacter spp.: } 7 \\
\text { E.coli: } 1 \\
\text { Klebsiella spp.: } 7\end{array}$ \\
\hline$\underset{23}{\text { Florescu DF et al/ Nebraska/ } 2012 .}$ & $98 / 146^{*}$ & $\begin{array}{l}\text { 96/146 (65,7\%) } \\
\text { S.aureus: } 13 \\
\text { MRSA: } 5 \\
\text { SCN: } 40 \\
\text { E.faecalis: } 33 \\
\text { E.faecium: } 17 \\
\text { VRE: } 10\end{array}$ & $\begin{array}{l}\text { 52/146 }(35,6 \%) \\
\text { Pseudomonas spp.: } 4 \\
\text { K.oxytoca: } 3 \\
\text { K.pneumoniae: } 18 \\
\text { Klebsiella spp. ESBL: } 5 \\
\text { E.coli: } 10 \\
\text { E.cloacae: } 16\end{array}$ \\
\hline $\begin{array}{l}\text { Rosado-Odom VM et al/ Indiana/ } \\
\text { 2011. }{ }^{21}\end{array}$ & $121 / 533^{\star *}$ & $\begin{array}{l}\text { 262/533 (40\%) } \\
\text { VRE: } 77 \\
\text { VSE: } 79 \\
\text { MSSA: } 12 \\
\text { MRSA: } 22 \\
\text { SCN: } 39 \\
\text { C.difficile: } 10 \\
\text { S.mitis: } 9 \\
\text { S.viridans } 3 \\
\text { S.salivarius: } 2 \\
\text { S.pyogenes: } 1 \\
\text { S.agalactiae: } 1 \\
\text { S.pneumoniae: } 1\end{array}$ & $\begin{array}{l}\text { 303/533 (47\%) } \\
\text { P.aeruginosa: } 80 \text { K.pneumoniae: } 69 \\
\text { E.coli: } 59 \\
\text { E.cloacae: } 40 \\
\text { K.oxytoca: } 17 \\
\text { A.baumannii: } 6 \\
\text { C.freundii: } 5 \\
\text { E.aerogenes: } 5 \\
\text { Enterobacter sp: } 5 \\
\text { S.maltophilia: } 4 \\
\text { P.mirabillis: } 3 \\
\text { S.marcescens: } 2 \\
\text { K.ascorbata: } 2 \\
\text { B.cepacea: } 2 \\
\text { A.xylos: } 1 \\
\text { B.fragilis: } 1 \\
\text { A.aerophila: } 1 / 533\end{array}$ \\
\hline $\begin{array}{l}\text { Primeggia J et al/ Washington/ } \\
2010 .{ }^{22}\end{array}$ & $28 / 35^{\star *}$ & $\begin{array}{l}\text { 14/35 (40\%) } \\
\text { Enterococcus spp.: } 5 \\
\text { Staphylococcus spp.: } 4\end{array}$ & $\begin{array}{l}17 / 35(50 \%) \\
\text { Pseudomonas spp.: } 6 \\
\text { E.coli: } 4 \\
\text { Klebsiella spp.: } 2\end{array}$ \\
\hline Kimura T et al/ Itália/ 2008. ${ }^{25}$ & $38 / 86^{* * *}$ & $\begin{array}{l}\text { 22/86 (25\%) } \\
\text { S.aureus: } 8 \\
\text { Enterococcus spp.: } 7 \\
\text { S.epidermidis: } 7\end{array}$ & $\begin{array}{l}\text { 52/86 (60\%) } \\
\text { P.aeruginosa: } 31 \\
\text { Klebsiella spp.: } 10 \\
\text { E.coli: } 11\end{array}$ \\
\hline John M et al. / Nova Iorque/ 2006. & $31 / 42^{\star *}$ & $\begin{array}{l}\text { 33/42 }(78,5 \%) \\
\text { MRSA: } 3 \\
\text { MSSA: } 1 \\
\text { SCN: } 14 \\
\text { VRE: } 5 \\
\text { VSE: } 8\end{array}$ & $\begin{array}{l}\text { 16/42 (38\%) } \\
\text { E.coli: ND } \\
\text { P.aeruginosa: ND } \\
\text { C.freundii: ND } \\
\text { K.pneumoniae: ND } \\
\text { E.cloacae: ND } \\
\text { S.marcescens: ND } \\
\text { H.influenza: ND } \\
\text { E.coli: ND } \\
\text { A.baumanii: ND }\end{array}$ \\
\hline Guaraldi G et al/ Itália/ 2005. ${ }^{20}$ & $19 / 100^{\star *}$ & $\begin{array}{l}\text { 19/100 (19\%) } \\
\text { S.epidermidis: } 13\end{array}$ & $\begin{array}{l}\text { 40/100 (40\%) } \\
\text { P.aeruginosa: } 24\end{array}$ \\
\hline
\end{tabular}

$\dagger$ Um paciente pode ter apresentado mais de um episódio de infecção

* Infecção de corrente sanguínea $\quad$ ** Infecções por agentes e em locais variados

***Infecção exclusivamente bacteriana ND: não disponível; GP: Gram-positivo; GN: Gram-negativo; MRSA: Staphylococcus aureus resistente a meticilina; MSSA: Staphylococcus aureus sensível a meticilina; VRE: Enterococcus resistente à vancomicina; VSE: Enterococcus sensível à vancomicina; SCN: Staphylococcus coagulase negativo 
Análise de dados de infecção da corrente sanguínea no TI observou que $65,9 \%$ foram causadas por Gram-positivos, e $34,1 \%$ por Gram-negativos. A distribuição por patógenos evidenciou maior número de casos de VRE, seguido por Sthaphylococcus coagulase negativo (SCN), Enterobacter spp. e Klebsiella spp., Pseudomonas spp., Streptococcus viridans (S.viridans), e, por fim, S.aureus e E.coli. ${ }^{5}$

Outro estudo realizado na Universidade de Nebraska incluiu 98 pacientes, sendo 21 TI e 77 TFI. Observaram que 68 pacientes apresentaram ao menos um episódio de infecção de corrente sanguínea, com um total de 146 episódios. As bactérias Gram-positivas foram responsáveis por 96 episódios (Staphylococcus aureus sensível a meticilina (MSSA) 8, MRSA 5, SCN 40 e Enterococcus spp. 48), Gram-negativas por 52 episódios (Pseudomonas spp. 4, Klebsiella spp. 21, E.coli 10, E.cloacae 16) e fungos 14 episódios (C.albicans 4, Candida parapsilosis (C.parapsilosis) 4, Candida glabrata (C.glabrata) 4 e espécies de Cryptococcus 2). Em relação ao tempo de ocorrência das infecções após o transplante, 26 episódios foram diagnosticados no primeiro mês, 74 nos primeiros três meses, 106 nos primeiros seis meses e 146 no primeiro ano. A incidência de infecção de corrente sanguínea foi maior nos pacientes com menor idade e a presença dessa infecção acarretou menor sobrevida. ${ }^{23}$

A infecção da corrente sanguínea é a mais comum, com taxas de 1,4 episódios por paciente na população pediátrica e 1,55 episódios por paciente entre adultos, após TI ou TMV. Corresponde a, aproximadamente, $60 \%$ das infecções bacterianas após o transplante. ${ }^{24}$

Outros sítios são também importantes no cenário das infecções bacterianas após TI e TMV. As infecções intraabdominais respondem por aproximadamente 13 a $37 \%$ das infecções bacterianas no período precoce pós-transplante, sendo os agentes mais comuns P.aeruginosa, Enterococcus spp, Enterobacter cloacae (E.cloacae) e E.coli. ${ }^{1}$

Em relação às infecções do trato respiratório, estas são responsáveis por 14 a $17 \%$ das infecções bacterianas no TI e no TMV, sendo que $39,5 \%$ das pneumonias relacionadas à assistência à saúde ocorrem nos primeiros dois meses. Os agentes mais frequentes são P.aeruginosa, K.pneumoniae, E.coli, Acinetobacter baumannii (A.baumannii) e Staphylococcus aureus (S.aureus). ${ }^{1}$

As infecções por MRSA vêm decrescendo na Europa e EUA, porém respondem por mais de $50 \%$ das infecções de corrente sanguínea em UTI. ${ }^{11}$ A principal fonte de S.aureus é a flora do próprio paciente (nasofaringe e pele) e o sítio de infecção, que por sua vez têm relação com procedimentos invasivos realizados (cirurgias, cateteres e intubação). ${ }^{11,26}$

Em um estudo de série de casos, a manifestação mais comum de infecção por S.aureus foi a infecção da corrente sanguínea $(45,7 \%)$, seguida de infecção pulmonar $(31,4 \%)$ e infecção do sítio cirúrgico (20\%). Desses, 60\% eram MSSA e $40 \%$ MRSA. Foram consideradas nosocomiais, 95\% das infecções, e a maioria das infecções recorrentes ocorreu em corrente sanguínea e pulmão. Quanto ao tempo entre o transplante e a primeira infecção, a média foi de 139 dias para infecção de corrente sanguínea, 266 dias para pulmonar e 41 dias para sítio cirúrgico, com a média total de 138 dias. As infecções tiveram uma distribuição temporal de $27 \%$ no primeiro mês, $45 \%$ até o terceiro mês e $63 \%$ até o sexto mês. A mortalidade após infecção pelo S.aureus em um mês e 12 meses foi de um (5\%) e seis (27\%), respectivamente. ${ }^{25,26}$ Nessa casuística, utilizou-se piperacilina-tazobactam como profilaxia cirúrgica por sete dias, e basiliximab ou timoglobulina associado ao tacrolimus e metilprednisolona como terapia de imunossupressão. Concluiu-se que cirurgias recentes, presença de cateter venoso central para NPT ou para hemodiálise e intubação foram fatores de risco para infecção pelo S.aureus. ${ }^{26}$

Estudos epidemiológicos de bactérias Gram-negativas MDR na população transplantada são limitados. A maioria das séries de casos é reportada em áreas endêmicas dessas bactérias, resultando em altas proporções de resistência, variando entre 18 a $50 \% .{ }^{12} \mathrm{O}$ risco de infecção de corrente sanguínea por bactérias Gram-negativas é 20 vezes maior nos pacientes submetidos a TOS em comparação com a população geral. ${ }^{22}$ Clinicamente, as bactérias mais importantes nesse cenário são as não fermentadoras como P.aeruginosa, Burkholderia spp., Stenotrophomonas maltophilia, Acinetobacter spp., além das Enterobactérias. ${ }^{12}$

Pseudomonas aeruginosa MDR e A.baumannii MDR estão em ascensão nas infecções pós-TOS, especialmente no período precoce. Essas infecções podem estar presentes desde o período pré-transplante, estando associadas a piores desfechos. No entanto, um relato de caso da Universidade de Nebraska concluiu que, se houver drogas ainda disponíveis, o TMV não está contra-indicado em caso de infecção por P.aeruginosa MDR. ${ }^{14,28}$

Infecção por Nocardia spp. vem aumentando nas últimas duas décadas, devido ao maior número de pacientes imunocomprometidos, mas também pelo aprimoramento das técnicas diagnósticas. A frequência dessa infecção nos TOS varia entre 0,7 e 3,5\%, sendo no TI $1,3 \%$. Geralmente ocorre no primeiro ano após o transplante, mas não é comum no primeiro mês. ${ }^{3}$

A apresentação mais comum da infecção por Nocardia é doença pulmonar, seguida de abscesso cerebral, meningite e doença disseminada. Os fatores de risco nos pacientes submetidos a TOS são altas doses de corticosteróides, história prévia de doença por CMV, altas doses de inibidores da calcineurina e hipogamaglobulinemia. Uma revisão de casos na Áustria identificou dois casos entre 24 pacientes submetidos a TI ou TMV. O primeiro caso 
ocorreu após três meses do TMV, com doença pulmonar, e o segundo, quatro semanas após o re-transplante, com abscessos hepáticos. ${ }^{3,29,30}$

A apresentação mais frequente da listeriose é a bacteremia, seguida da meningoencefalite, e os fatores de risco para sua ocorrência são diabetes mellitus, infecção por CMV e altas doses de corticosteróides. O uso de sulfametoxazoltrimetoprim tem efeito protetor. ${ }^{3}$

Uma das principais causas de diarreia nos indivíduos submetidos a TOS é infecção por C.difficile. Sua incidência no TI é de 9\%. Uma revisão de casos da Áustria analisou a epidemiologia das infecções pelo C.difficile, e observou-se que, dos 26 casos de TI ou TMV, dois apresentaram essa comorbidade. ${ }^{3,31,32}$

A epidemiologia dessas infecções tem se modificado devido às profilaxias instituídas, à sofisticação dos agentes imunossupressores e às melhores técnicas diagnósticas. ${ }^{3} \mathrm{O}$ uso de esquemas antibióticos de maior espectro, adjunto à exposição nosocomial, propiciou a emergência de agentes multi-droga resistentes (MDR). ${ }^{33,34}$ É determinado por fatores como exposição epidemiológica e um complexo de características que envolvem o risco individual de infecção, "estado de equilíbrio da imunossupressão". $\mathrm{O}$ desenvolvimento das profilaxias e o ajuste da imunossupressão podem alterar esse quadro. ${ }^{2,3,35}$

No entanto, coleções pós-operatórias, uso de dispositivos invasivos, uso de drenos, isquemia tecidual, neutropenia, doenças crônicas associadas, disfunção orgânica, distúrbios metabólicos, exposição antimicrobiana, entre outros, também contribuem para o risco de adquirir infecção. ${ }^{2,3,35}$
Alguns fatores de risco já estão bem estabelecidos na literatura: cirurgias recorrentes, mudança da flora nosocomial (resistência antimicrobiana), hospitalização prolongada pré-transplante, imunossupressão intensa, uso de dispositivos invasivos, infecção proveniente do doador e exposição epidemiológica. ${ }^{36}$

\section{CONCLUSÃO}

O risco de infecção varia conforme o tempo de progressão após o transplante, tipo e intensidade de imunossupressão e grau de exposição aos diferentes micro-organismos. $\mathrm{O}$ estado de equilíbrio da imunossupressão é um risco amplamente determinado pela dose, duração e sequência da terapia imunossupressora. Os fatores individuais de risco devem ser considerados para escolha da estratégia profilática e terapia empírica, e para determinação dos diagnósticos diferenciais de cada infecção.

Assim, os agentes mais prevalentes nos transplantes de intestino e multiviscerais foram: Enterococcus spp., Staphylococcus spp., Streptococcus spp., Pseudomonas aeruginosa, Klebsiella pneumoniae, Escherichia coli, Enterobacter spp. Os principais sítios de infecção foram: sítio cirúrgico, intra-abdominal, corrente sanguínea, cateter venoso central, urinário e respiratório. Os fatores de risco identificados foram: rejeição aguda, dose alta de imunossupressão, uso de dispositivos invasivos, cirurgias extensas e reoperação, enxerto contaminado, infecção prévia e paciente hospitalizado no período pré-transplante.

\section{ABSTRACT}

The nature of infections after solid organ transplantation has changed significantly upon the advent of potent immunosuppressive regimens, the routine use of antibiotic prophylaxis and the advancement of microbiological diagnostic techniques. New pathogens are being identified in this population, including several with a significant antimicrobial resistance profiling. The after-transplant chronology of infections is set by the nature and intensity of individual epidemiological exposure and the immunosuppressant state of balance. The shortage of epidemiological studies on bowel and multivisceral post-transplant infections still keep their trend of being more a state of the art understanding than as science. The purpose of this study is to describe epidemiological data of intestine bacterial and multivisceral posttransplant infections. The most prevalent agents in the Small bowel and Multivisceral transplants were: Enterococcus spp, Staphylococcus spp, Streptococcus spp, Pseudomonas aeruginosa, Klebsiella pneumoniae, Escherichia coli, Enterobacter spp. The main sites of infection were the surgical site, intra-abdominal, blood, central venous catheter, urinary and respiratory systems. The identified risk factors were: acute rejection, high dose of immunosuppression, use of invasive devices, extensive surgery and reoperation, contaminated graft, previous infection and hospitalized patients in the pretransplant period.

Keywords: Bacterial Infections; Intestine, Small; Transplantation. 


\section{REFERÊNCIAS}

1. Timpone Jr JG, Girlanda R, Rudolph L, Fishbein TM. Infections in intestinal and multivisceral transplant recipients. Infect Dis Clin North Am. 2013 Jun;27:359-77.

2. Fishman JA, Issa NC. Infection in organ transplantation: risk factors and evolving patterns of infection. Infect Dis Clin North Am. 2010;24:273-83.

3. Muñoz P, Fernández NS, Fariñas MC. Epidemiology and risk factors of infections after solid organ transplantation. Enferm Infecc Microbiol Clin. 2012;30(Supl 2):10-8.

4. Grim SA, Clark Nm. Management of infectious complications in solid organ transplant recipients. Clin Pharmacol Ther. 2011;90:333-42.

5. Akhter K, Timpone J, Matsumoto C, Fishbein T, Kaufman S, Kumar P. Six-month incidence of bloodstream infections in intestinal transplant patients. Transpl Infect Dis. 2012;14:242-7.

6. Goulet O, Sauvat F, Ruemmele F, Caldari D, Damotte D, Cezard JP, Lacaille F, Canioni D, Hugot JP, Berebi D, Sarnacki S, Colomb V, Jan D, Aigrain Y, Revillon Y. Results of the Paris program: ten years of pediatric intestinal transplantation. Transpl Proc. 2005;37:1667-70.

7. Abu-Elmagd K, Costa G, Bond GJ, Soltys K, Sindhi R, Wu T, Koritsky DA, Schuster B, Martin L, Cruz RJ, Murase N, Zeevi A, Irish W, Ayyash MO, Matarese L,Humar A, Mazariegos G. Five hundred intestinal and multivisceral transplantations at a single center. Ann Surg. 2009;250:567-81.

8. Kato T, Tzakis AG, Selvaggi G, Gaynor JJ, David AI, Bussotti A, Moon JI, Ueno T, DeFaria W, Santiago S, Levi DM, Nishida S, Velasco ML, McLaughlin G,Hernandez E, Thompson JF, Cantwell P, Holliday N, Livingstone AS, Ruiz P. Intestine and multivisceral transplantation in children. Ann Surg. 2006;243:756-64.

9. Farmer DG, Venick RS, Colangelo J, Esmailian Y, Yersiz H, Duffy JP, Cortina GR, Artavia K, Ngo K, McDiarmid SV, Busuttil RW. Pretransplant predictors of survival after intestinal transplantation: analysis of a single-center experience of more than 100 transplants. Transplantation. 2010;90:1574-80.

10.Fishman JA and the AST Infectious Diseases Community of Practice. Introduction: infection in solid organ transplant recipients. Am J Transpl. 2009;9(Suppl 4):S3-S6.

11.Garzoni C. Methicillin-Resistant, vancomycin-intermediate and vancomycin-resistant staphylococcus aureus infections in solid organ transplantation. Am J Transpl. 2013 Mar;13:50-8.

12.van Duin D, van Delden C; AST Infectious Diseases Community of Practice. Multidrug-resistant gram-negative bacteria infections in solid organ transplantation. Am J Transpl. 2013 Mar;13:31-41.

13. Palocaren MS. An overview of intestine and multivisceral transplantation. Crit Care Nurs Clin Norht Am. 2011;23:457-69.

14.Cicalese L, Sileri P, Green M, Abu-Elmagd K, Kocoshis S, Reyes J. Bacterial translocation in clinical intestinal transplantation. Transplantation. 2001;71:1414-7.

15.Soin B. Multivisceral abdominal transplantation. Curr Opin Organ Transpl. 2002;7:208-13.

16.John M, Gondolesi G, Herold BC, Kaufman S, Fishbein T, Posada R. Impact of surveillance stool culture guided selection of antibiotics in the management of pediatric small bowel transplant recipients. Pediatr Transpl. 2006:10:198-204.
17. Moreno A, Cervera C, Gavaldá J, Rovira M, de la Cámara R, Jarque I et al. Bloodstream infections among transplant recipients: results of a nationwide surveillance in Spain. Am J Transpl. 2007;7:257986.

18.Tzakis AG, Kato T, Levi DM, Defaria W, Selvaggi G, Weppler D, et al. 100 Multivisceral transplants at a single center. Ann Surg. 2005;242:480-93.

19.Zanfi C, Lauro A, Cescon M, Dazzi A, Ercolani G, Grazi GL, et al. Daclizumab and alemtuzumab as induction agents in adult intestinal and multivisceral- transplantation: rejection and infection rates in 40 recipients during the early postoperativeperiod. Transplant Proc. 2010;42:35-8.

20.Guaraldi G, Cocchi S, Codeluppi M, Di Benedetto F, De Ruvo N, Masetti M, et al. Outcome, incidence and timing of infectious complications in small bowel and multivisceral organ transplantation patients. Transplantation. 2005;80:1742-8.

21.Rosado-Odom VM, Johnson R, Mangus RS. Bacterial, fungal, and parasitic infections one year after isolated intestinal and multivisceral transplantation in a single centersession: Poster Abstract Session: Infections after solid organ transplants. Annual Meeting IDSA; Boston; 2011

22.Primeggia J, Timpone JG, Karacki P, Matsumoto C, Fishbein T. Infection among small bowel and multivisceral transplant recipients in the 30-day post-operative period. session: Abstracts: Transplant ID. Annual Meeting, 48, Vancouver; 2010.

23.Florescu DF, Qiu F, Langnas AN, Mercer DF, Chambers H, Hill LA et al. Bloodstream infections during the first year after pediatric small bowel transplantation. Pediatr Infect Dis J. 2012;31:700-4.

24. Reyes JD, Green M. Risks and Epidemiology of Infections after Intestinal Transplantation. Bowden RA, Ljungman P, Snydman DR, editores. Transplant Infections. Philadelphia: Lippincott Williams \& Wilkins; 2010:179-86

25.Kimura T, Lauro A, Cescon M, Zanfi C, Dazzi A, Ercolani G et al. Impact of induction therapy on bacterial infections and long-term outcome in adult intestinal and multivisceral transplantation: a comparison of two different induction protocols: daclizumab vs. alemtuzumab. Clin Transplant. 2009;23:420-5.

26.Florescu D, Qiu F, Vivekanandan R, Mercer DF, Langnas AN, Kalil AC. Risk factors and outcomes of Staphylococcus aureus infections after small bowel and multivisceral transplantation. Pediatr Infect Dis J. 2012;31:25-9

27.Al-Hasan MN, Razonable RR, Eckel-Passow JE, Baddour LM. Incidence rate and outcome of gram-negative bloodstream infection in solid organ transplant recipients. Am J Transpl. 2009;9:835-43.

28.Florescu D, Grant W, Botha JF, Fey P, Kalil AC. Should multivisceral transplantation be considered in patients colonized with multidrug-resistant pseudomonas aeruginosa? Microb Drug Resist. 2012;18:74-8

29.Clark NM, Reid GE; AST Infectious Diseases Community of Practice. Nocardia infections in solid organ transplantation. Am J Transpl. 2013;13:83-92.

30.Wiesmayr S, Stelzmueller I, Tabarelli W, Bargehr D, Graziadei I, Freund $\mathrm{M}$ et al. Nocardiosis following solid organ transplantation: a single-centre experience. Transpl Int. 2005;18:1048-53. 
31.Dubberke ER, Burdette SD; AST Infections Diseases Community of Practice. Clostridium difficile infections in solid organ transplantation. Am J Transpl. 2013;13:42-9.

32. Stelzmueller I, Goegele H, Biebl M, Wiesmayr S, Berger N, Tabarelli $\mathrm{W}$ et al. Clostridium difficile colitis in solid organ transplantation - a single-center experience. Dig Dis Sci. 2007;52:3231-6.

33.Ison MG, Stosor V. Transplantation of high-risk donor organs: a survey of US solid organ transplant center as practices as reports by transplant infectious disease physicians. Clin Transpl. 2009:23:866-73.
34.Garg M, Jones RM, Vaughan RB, Testro AG. Intestinal transplantation: Current status and future directions. J Gastroenterol Hepatol. 2011;26:1221-8.

35.Fishman JA and the AST Infectious Diseases Community of Practice. Introduction: infection in solid organ transplant recipients. Am J Transpl. 2009;9(Suppl 4):S3-S6.

36. Oltean M, Herlenius G, Gäbel M, Friman V, Olausson M. Infectious complications after multivisceral transplantation in adults. Transpl Proc. 2006;38:2683-5. 